Supporting information for

\title{
Effective Capture of Cefradines in Water with a Highly Stable Zr(IV)-Based Bimetal-Organic Framework
}

Yitian Zhou, Le Yu, Yuan Gao, Jiafei Wu, and Wei Dai*

College of Chemistry and Life Science, Zhejiang Normal University, Zhejiang Province Jinhua 321004, People's Republic of China

\#Le Yu equally contributed to this work as Yitian Zhou, and they are both the first author.<smiles>CC1=C(C(=O)O)N2C(=O)[C@@H](NC(=O)[C@H](N)C3=CCC=CC3)[C@H]2SC1</smiles>

Scheme S1. The molecule structure of cefradines (molecule size: $1.3 \times 0.6 \times 0.6 \mathrm{~nm}$ ).

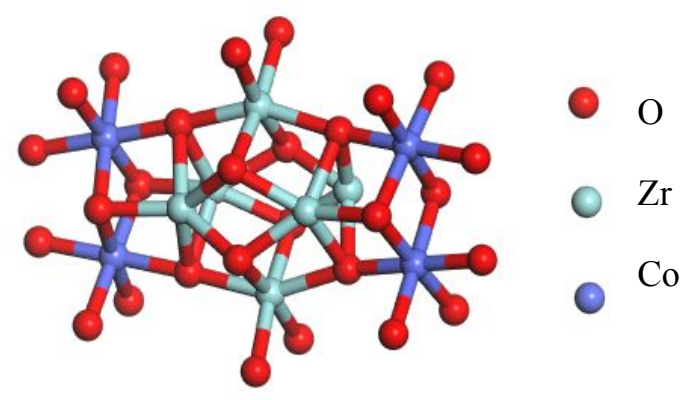

Scheme S2. $\mathrm{Zr}_{6}-\mathrm{Co}_{4}$ bimetallic cluster. 
Table S1. Different isotherm models and their linear forms.

\begin{tabular}{cccc}
\hline Isotherm & Nonlinear form & Linear form & Plot \\
\hline Langmuir & $q_{e}=\frac{K_{L} C_{e}}{1+K_{L} C_{e}} \quad \frac{C_{e}}{q_{e}}=\frac{1}{q_{L} \cdot K_{L}}+\left(\frac{1}{q_{L}}\right) \cdot C_{e}$ & $\frac{C_{e}}{q_{e}}$ versus $C_{e}$ \\
Freundlich & $q_{e}=K_{f} C_{e}^{\frac{1}{n}}$ & $\ln q_{e}=\ln K_{f}+\left(\frac{1}{n}\right) \cdot \ln C_{e} \quad \ln q_{e}$ versus $\ln C_{e}$
\end{tabular}

Where $q_{e}$ is the equilibrium adsorption capacity in $\mathrm{mg} / \mathrm{g} ; K_{L}$ is a constant related to the affinity of the binding sites in $\mathrm{L} / \mathrm{mg}$; ' $K$ ' and ' $n$ ' are measures of adsorption capacity and the intensity of adsorption respectively.

Table S2. Calculation equations.

\begin{tabular}{cl}
\hline Name & \multicolumn{1}{c}{ Equations } \\
\hline Pseudo-first order model & $\ln \left(q_{e}-q_{t}\right)=\ln \left(q_{e}\right)-K_{1} t$ \\
Pseudo-second order model & $\frac{t}{q_{t}}=\frac{1}{K_{2} q_{e}}+\frac{t}{q_{e}}$ \\
Intra-particle diffusion model & $q_{t}=K_{3} t^{1 / 2}$ \\
\hline
\end{tabular}

Where $q_{e}$ and $q_{t}(\mathrm{mg} / \mathrm{g})$ are the uptakes of thiophene at equilibrium and at time $t$ (min), respectively, $K_{l}(1 / \mathrm{min})$ is the adsorption rate constant, $K_{2}(\mathrm{~g} / \mathrm{mg}$.min) is the rate constant for the second-order equation, and $K_{3}\left(\mathrm{mg} / \mathrm{g} \cdot \mathrm{min}^{1 / 2}\right)$ is the intra-particle diffusion rate constant.

Where $q_{e, e x p}$ and $q_{e, c a l}$ are the experimental and calculation uptakes of adsorbates, respectively.

Table S3. Structural properties and metal ( $\mathrm{Zr}$ and $\mathrm{Co}$ ) contents of UiO-66, UiO-66-Co, UiO-67 and UiO-67-Co, respectively.

\begin{tabular}{ccccccc}
\hline Samples & $\begin{array}{c}\mathrm{S}_{\mathrm{BET}} \\
\left(\mathrm{m}^{2} / \mathrm{g}\right)\end{array}$ & $\begin{array}{c}\mathrm{V}_{\text {total }} \\
\left(\mathrm{cm}^{3} / \mathrm{g}\right)\end{array}$ & $\begin{array}{c}\mathrm{V}_{\text {mic }} \\
\left(\mathrm{cm}^{3} / \mathrm{g}, \%\right)\end{array}$ & $\begin{array}{c}\text { Average pore } \\
\text { diameter }(\mathrm{nm})\end{array}$ & $\begin{array}{c}\mathrm{Zr} \text { contents } \\
(\mathrm{wt} \%)^{\mathrm{a}}\end{array}$ & $\begin{array}{c}\text { Co contents } \\
(\mathrm{wt} \%)^{\mathrm{a}}\end{array}$ \\
\hline UiO-66 & 1411 & 0.70 & 75 & 2.03 & 48.29 & 0 \\
UiO-66-Co & 1326 & 0.57 & 70 & 2.19 & 41.35 & 8.12 \\
UiO-67 & 1984 & 0.92 & 82 & 1.45 & 45.67 & 0 \\
UiO-67-Co & 1637 & 0.67 & 78 & 1.79 & 36.23 & 7.55 \\
\hline
\end{tabular}

${ }^{\mathrm{a}} \mathrm{Zr}$ and Co contents were analyzed by ICP-OES. 
Table S4. Comparison of the maximum uptake capacities of cefradine on various adsorbents.

\begin{tabular}{ccc}
\hline Adsorbents & Maximum uptake capacities $(\mathrm{mg} / \mathrm{g})$ & References \\
\hline Peanut husk & 36 & {$[37]$} \\
CdS-MWCNT & 41 & {$[38]$} \\
PCN-222 & 333 & {$[5]$} \\
MIL-101-NH 2 -Cr & 70 & {$[5]$} \\
Wheat straw & 46 & {$[39]$} \\
Activated carbon & 123 & {$[40]$} \\
Biochar & 62 & {$[41]$} \\
UiO-66-Co & 975 & This work \\
UiO-67-Co & 1091 & This work \\
\hline
\end{tabular}

Table S5. Parameters of the isotherm models for the adsorption processes of cefradine onto Zr-Co MOFs.

\begin{tabular}{ccccccc}
\hline \multirow{2}{*}{ Samples } & \multicolumn{3}{c}{ Langmuir } & \multicolumn{3}{c}{ Freundlich } \\
& $q_{e}(\mathrm{mg} / \mathrm{g})$ & $K_{\mathrm{L}}(\mathrm{L} / \mathrm{mg})$ & $R^{2}$ & $K_{\mathrm{f}}(\mathrm{L} / \mathrm{g})$ & $n$ & $R^{2}$ \\
\hline UiO-66 & 620 & 17.3 & 0.9840 & 24.5 & 1.35 & 0.9248 \\
UiO-66-Co & 770 & 43.1 & 0.9930 & 156.9 & 2.69 & 0.9310 \\
UiO-67 & 971 & 97.0 & 0.9725 & 117.3 & 1.69 & 0.9248 \\
UiO-67-Co & 1200 & 51.8 & 0.9748 & 85.3 & 1.49 & 0.9575 \\
\hline
\end{tabular}

Table S6. Parameters of the kinetic models for the adsorption processes of cefradine onto Zr-MOFs.

\begin{tabular}{cccccccccc}
\hline & \multicolumn{3}{c}{ Pseudo-first-order rate equation } & \multicolumn{3}{c}{$\begin{array}{c}\text { Pseudo-second-order rate } \\
\text { equation }\end{array}$} & \multicolumn{3}{c}{ Intra-particle diffusion model } \\
\cline { 2 - 10 } Samples & $\begin{array}{c}q_{e, \exp } \\
(\mathrm{mg} / \mathrm{g})\end{array}$ & $\begin{array}{c}K_{l} \\
(1 / \mathrm{min})\end{array}$ & $R^{2}$ & $\begin{array}{c}q_{e, \exp } \\
(\mathrm{mg} / \mathrm{g})\end{array}$ & $\begin{array}{c}K_{2}(\mathrm{~g} / \mathrm{mg} \\
\mathrm{min})\end{array}$ & $R^{2}$ & $\begin{array}{c}q_{e, \exp } \\
(\mathrm{mg} / \mathrm{g})\end{array}$ & $\begin{array}{c}K_{3}(\mathrm{mg} / \mathrm{g} \\
\left.\mathrm{min}^{1 / 2}\right)\end{array}$ & $R^{2}$ \\
\hline UiO-66 & 608 & 0.0144 & 0.6994 & 608 & 117.1 & 0.9997 & 608 & 9.35 & 0.5793 \\
UiO-66-Co & 756 & -0.0007 & 0.5079 & 756 & 204.3 & 0.9997 & 756 & 8.04 & 0.6768 \\
UiO-67 & 978 & 0.0107 & 0.4149 & 978 & 158.8 & 0.9995 & 978 & 16.35 & 0.6957 \\
UiO-67-Co & 1086 & -0.0132 & 0.1939 & 1086 & 75.25 & 0.9999 & 1086 & 30.00 & 0.7860 \\
\hline
\end{tabular}



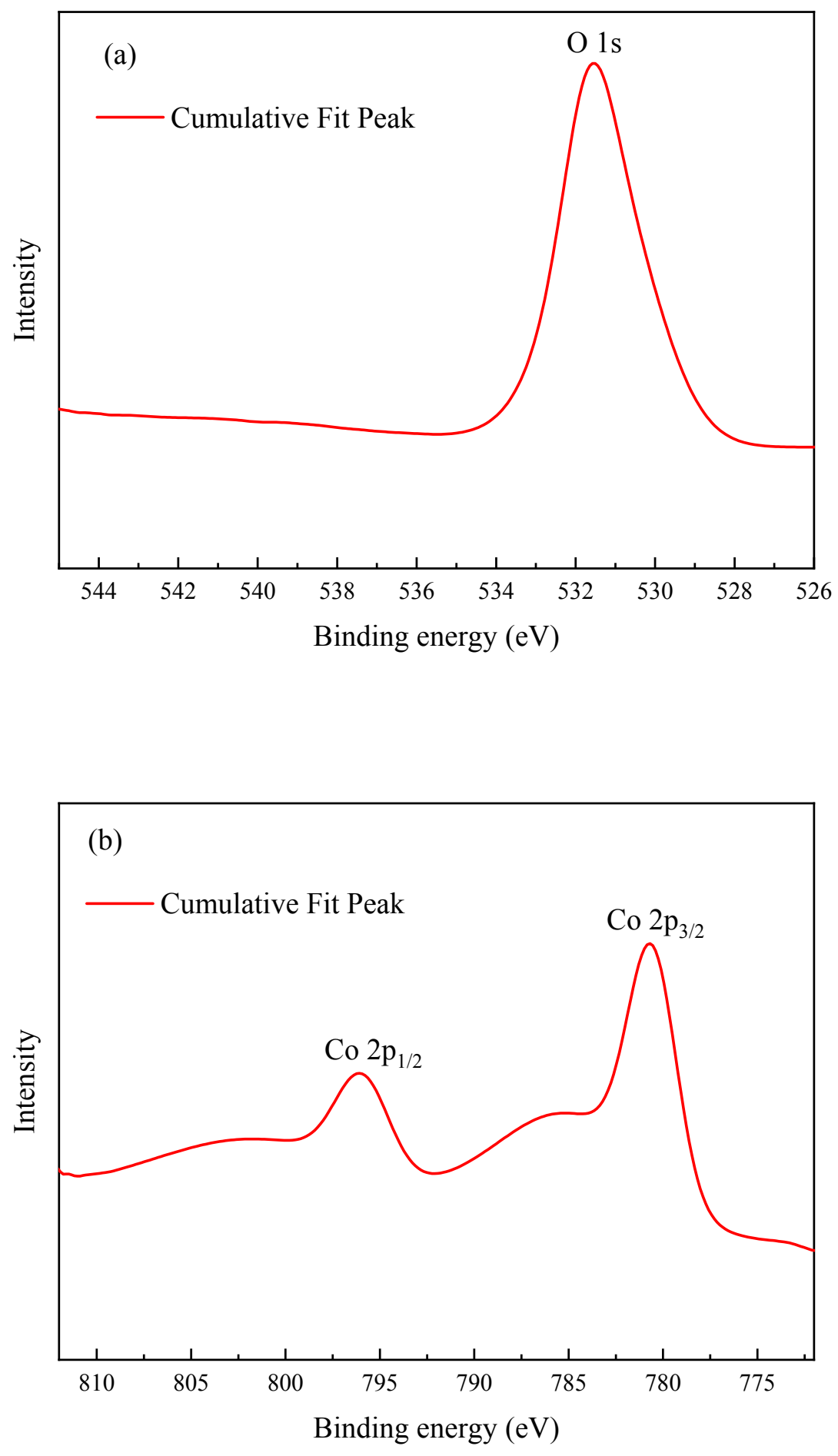

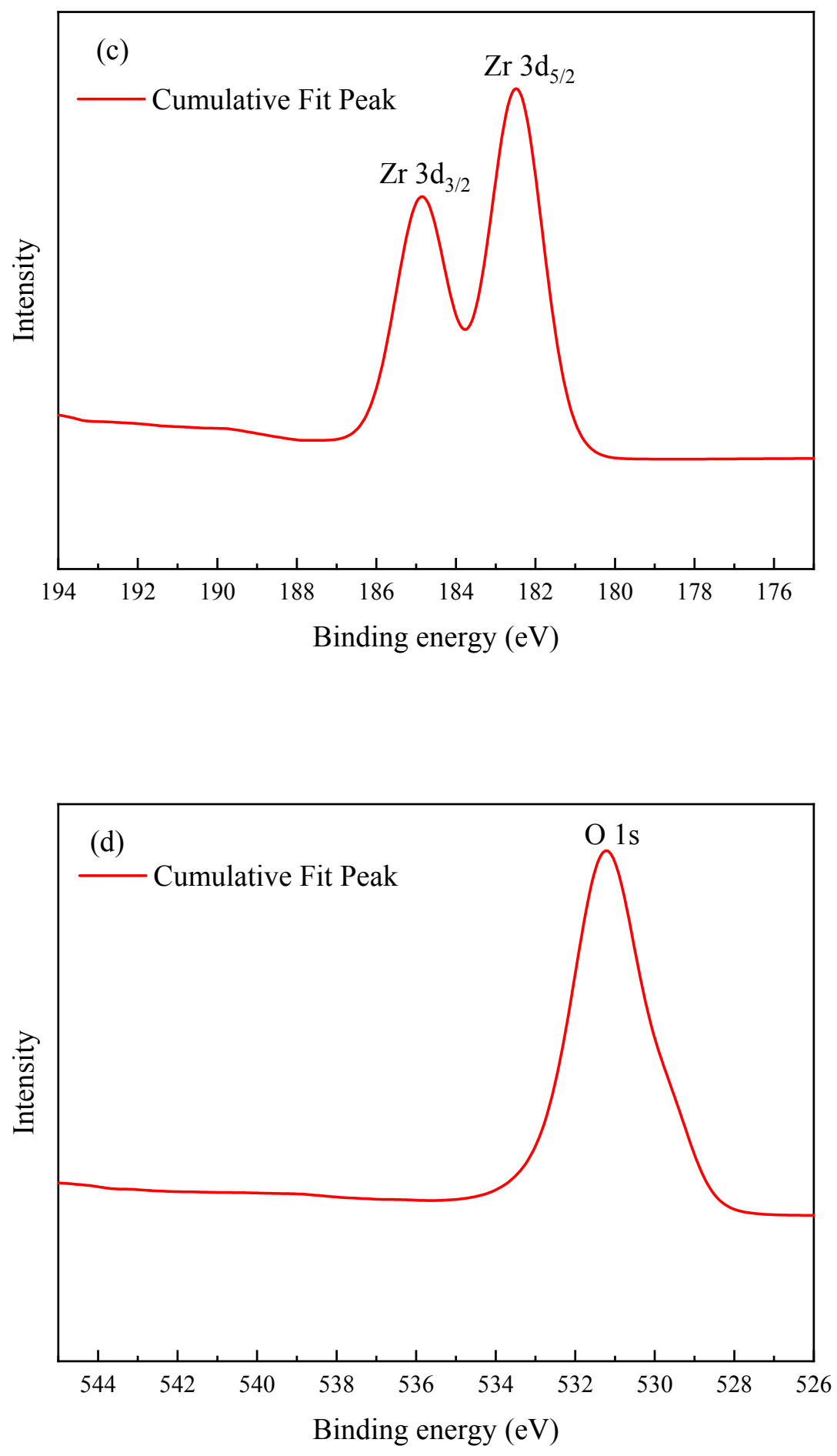

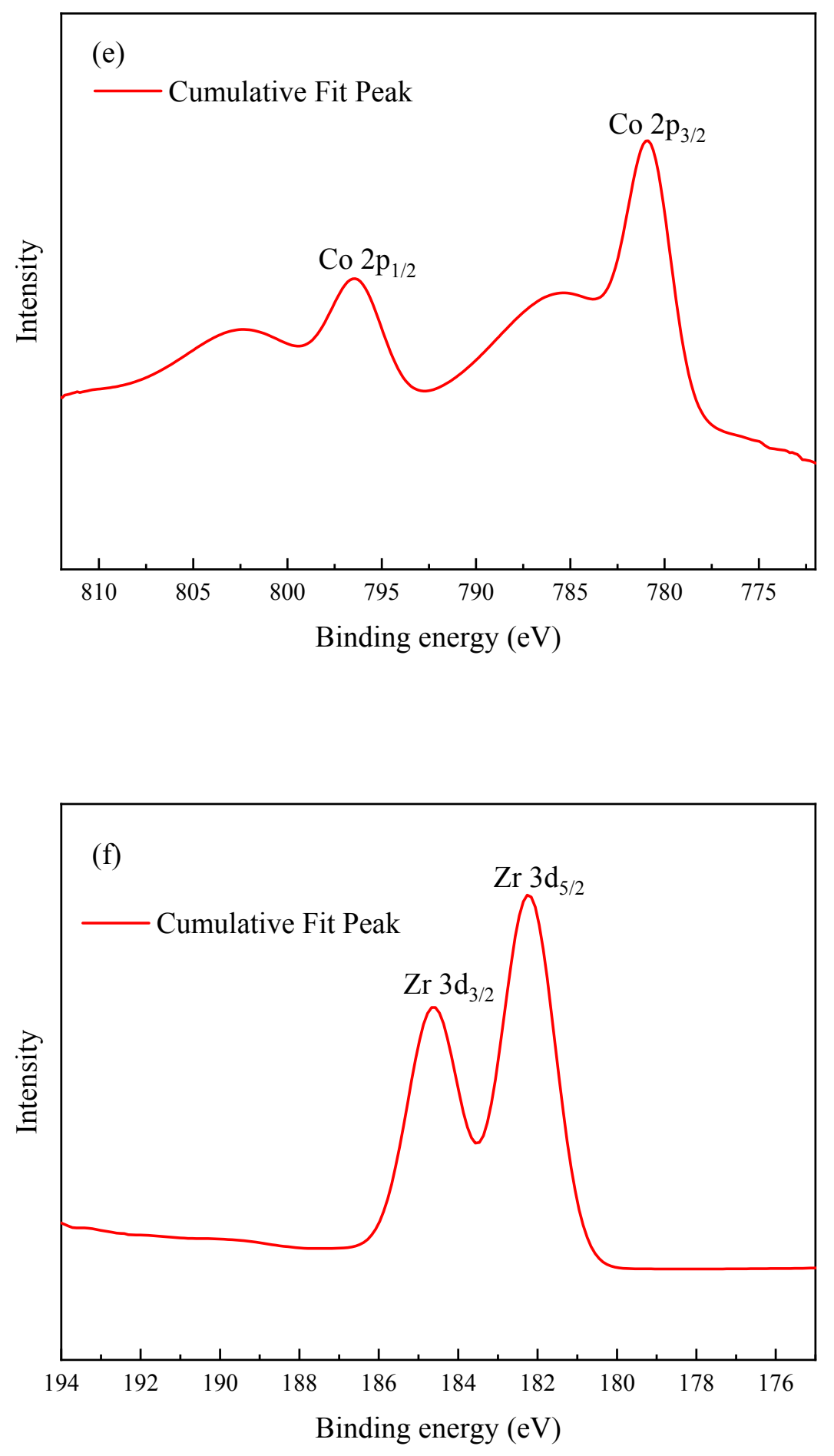

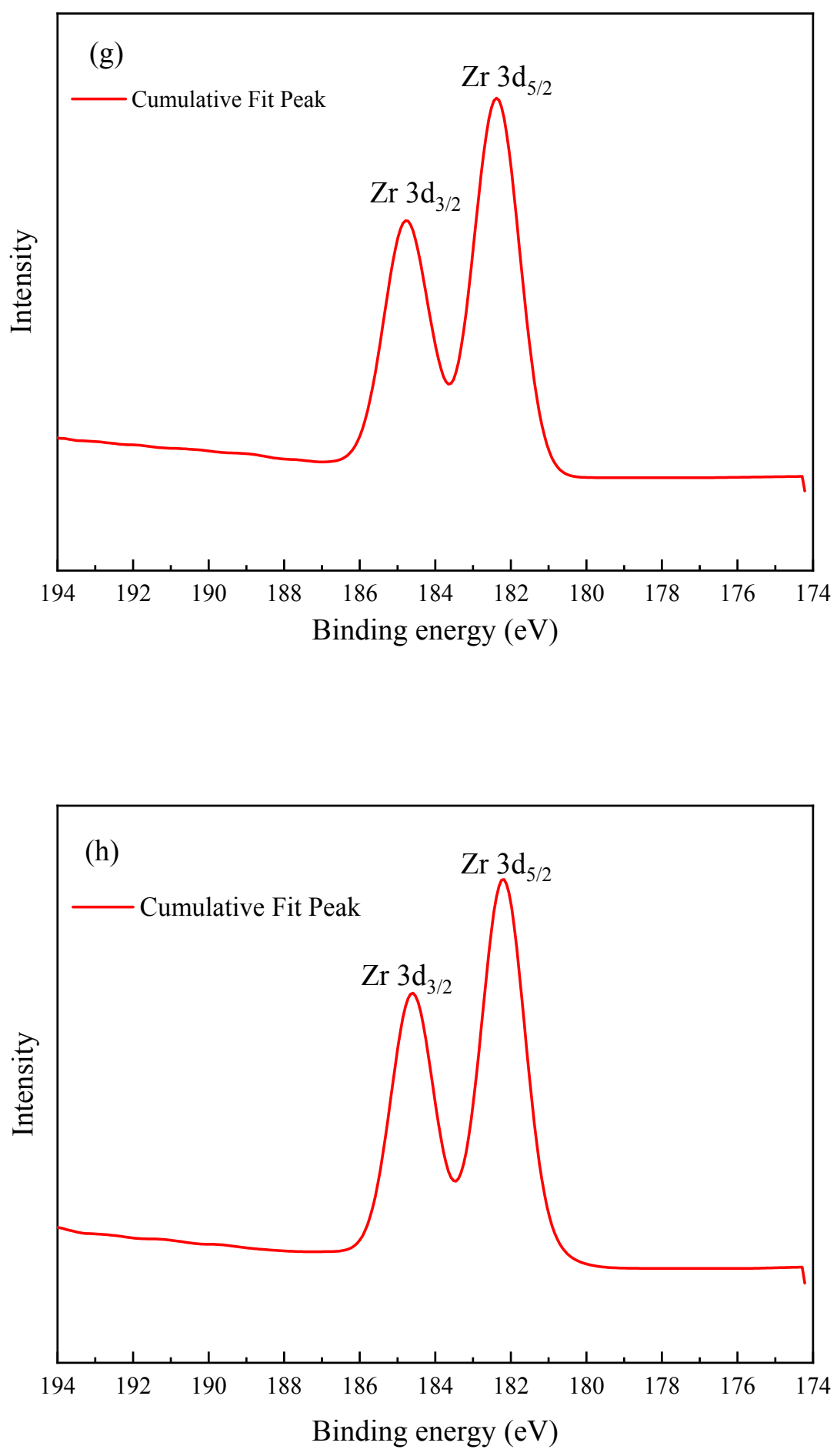

Figure S1. XPS results of Zr-MOFs in which (a), (b), (c) represent UIO-66-Co, (d), (e), (f) represent UIO-67-Co and (g), (h) represent UIO-66 and UIO-67, respectively. *Elemental symbols applied in the graphs. 


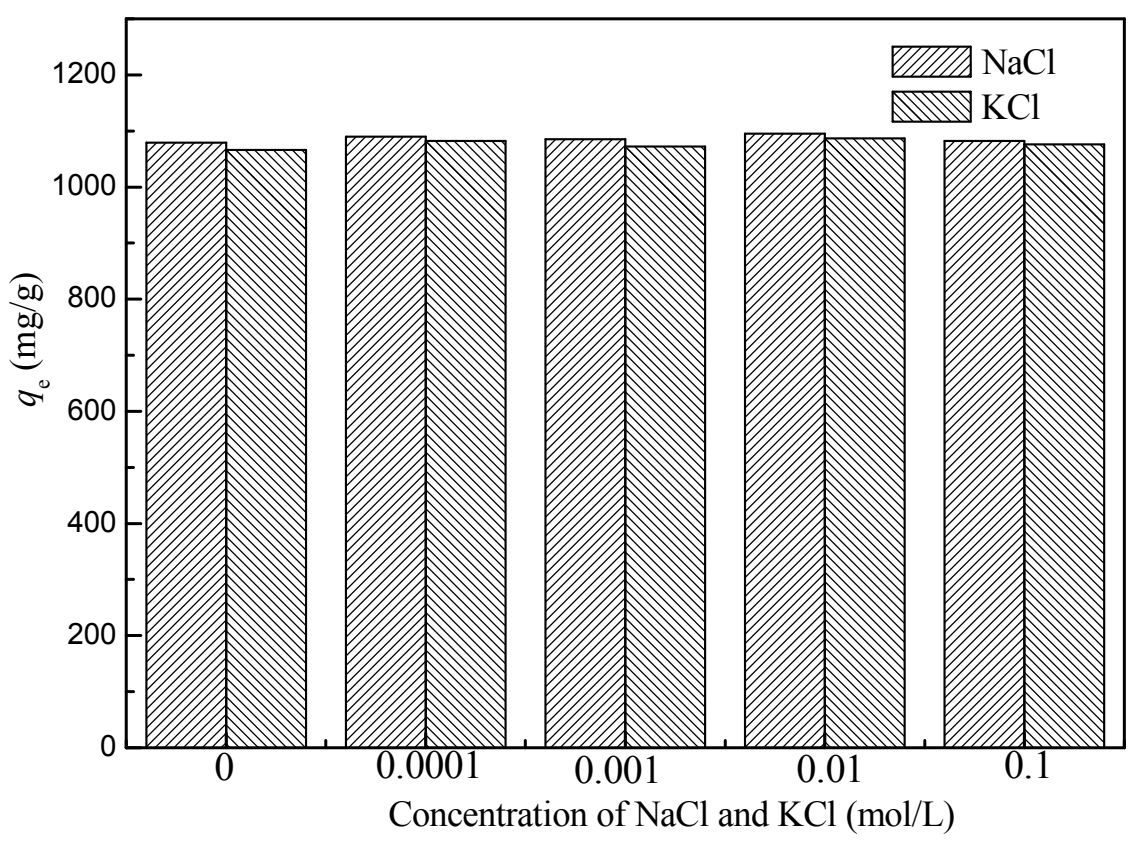

Figure S2. Adsorption capacity of cefradine with different concentrations of coexisting ions.

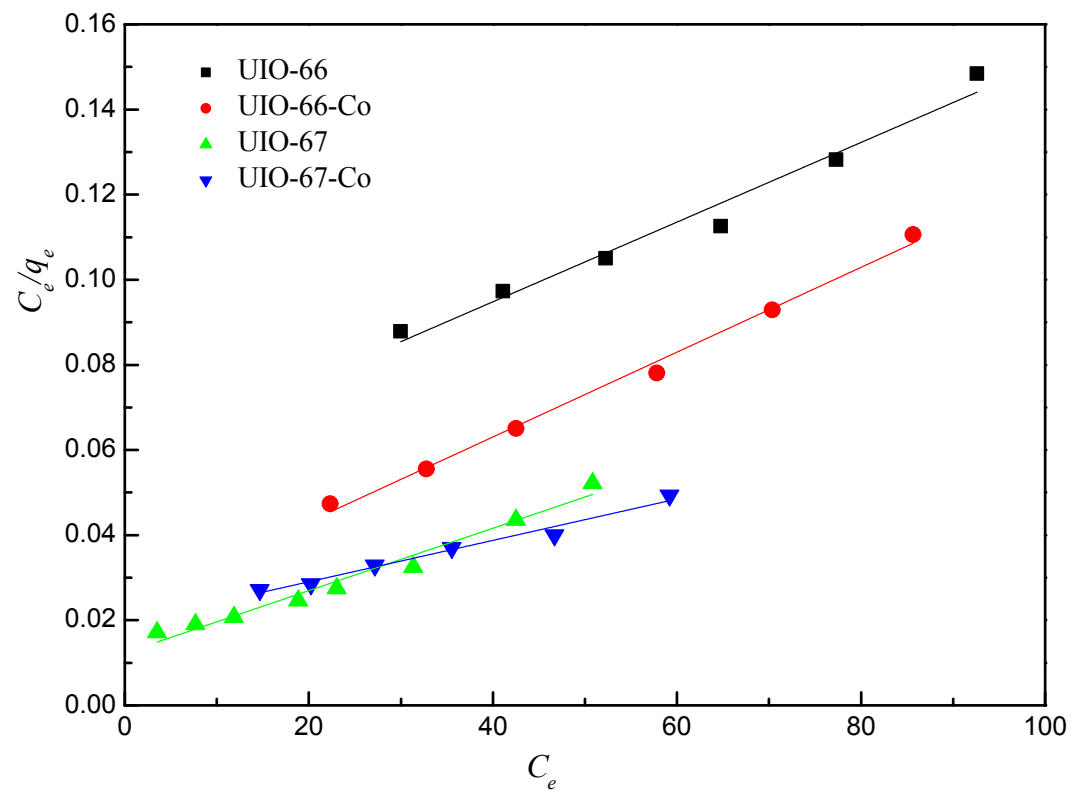

Figure S3. Langmuir fitting of the adsorption process of cefradine onto MOFs. 


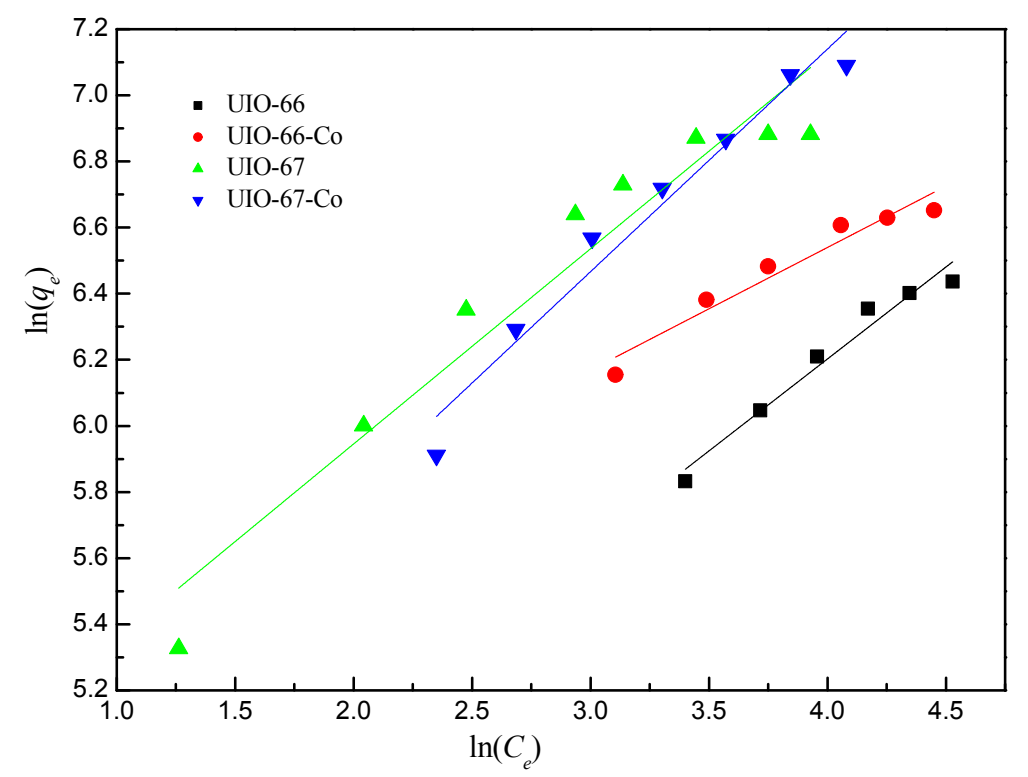

Figure S4. Freundlich fitting of the adsorption process of cefradine onto MOFs

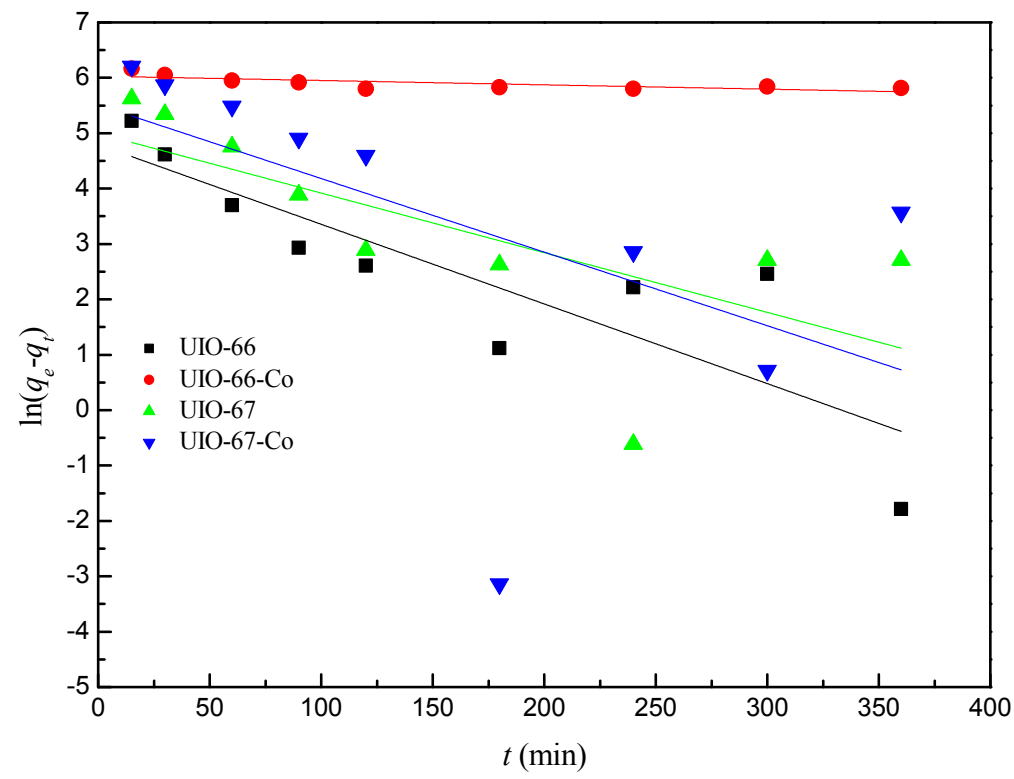

Figure S5. Pseudo-first fitting of the adsorption kinetics of cefradine onto MOFs 


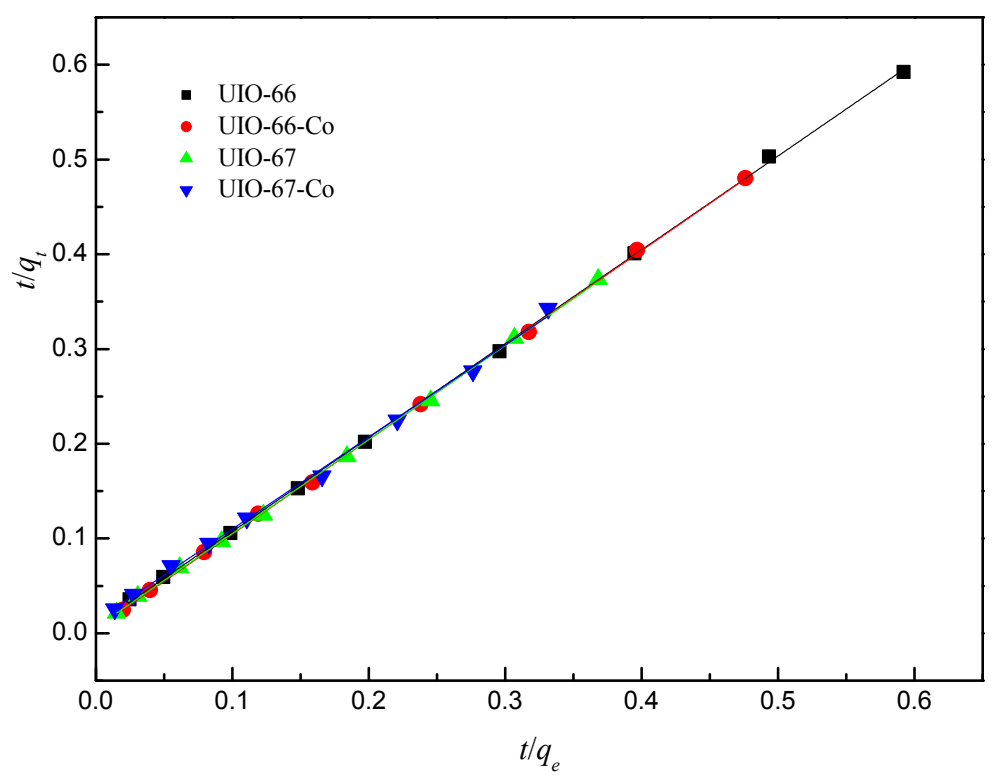

Figure S6. Pseudo-second fitting of the adsorption kinetics of cefradine onto MOFs

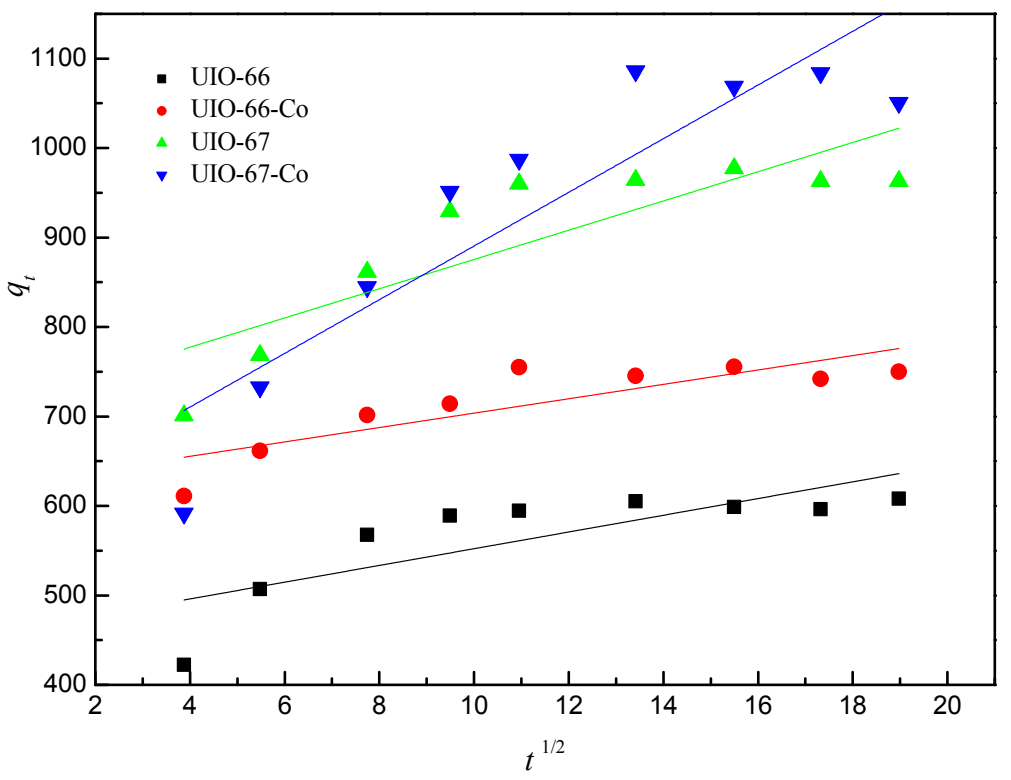

Figure S7. Intra-particle diffusion fitting of the adsorption kinetics of cefradine onto MOFs. 

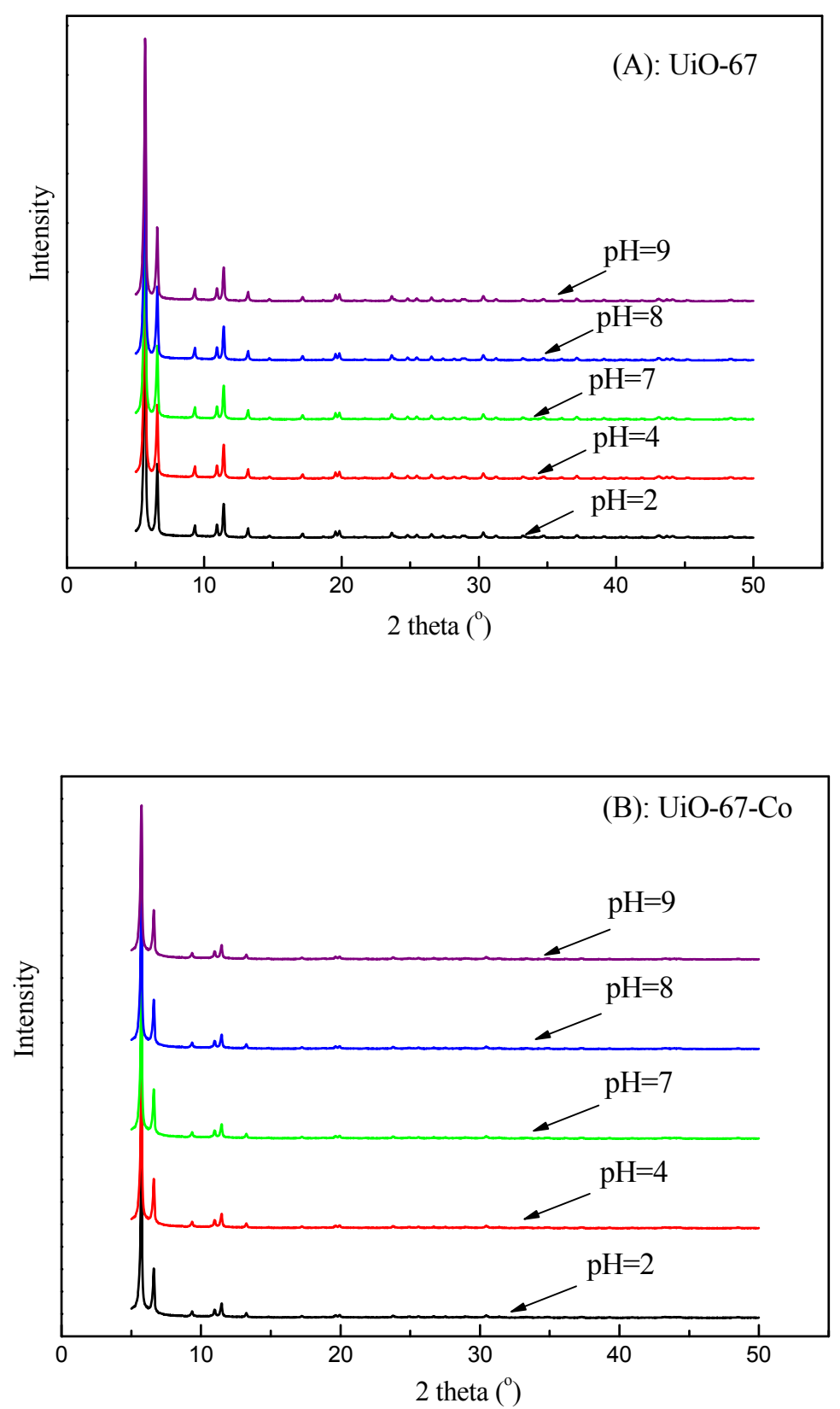

Figure S8. PXRD patterns of UiO-67 and UiO-67-Co after water stability tests (pH=2, 4, 7, 8, and 9). (A): UiO-67; (B): UiO-67-Co. 